\title{
Qualitative strain elastography - strain ratio evaluation - an important tool in breast cancer diagnostic
}

\author{
Dana Stoian', Bogdan Timar², Marius Craina1, Elena Bernad', Izabela Petre', \\ Mihaela Craciunescu ${ }^{3}$
}

${ }^{1}$ Department of Obstetrics Gynecology, ${ }^{2}$ Department of Medical Informatics, ${ }^{3}$ Department of Microbiology, University of Medicine and Pharmacy "Victor Babes" Timisoara, Romania

\begin{abstract}
Aims: To assess the quality of real time elastography as a differential diagnosis tool for breast nodules and to compare it with standard ultrasonography. Material and methods: In this prospective study we enrolled 174 patients in which 174 dominant breast nodules were considered for the final diagnosis. The results of ultrasonography and real time elastography, both qualitative and quantitative, were compared with pathology findings from the biopsy specimens. Results: Pathology examinations determined 102 nodules were benign and 72 malignant. Qualitative elastography had a better diagnostic performance (82.4\% sensitivity and $81.9 \%$ specificity) than ultrasonography plus Doppler evaluation $(70.3 \%$ sensitivity and $73.5 \%$ specificity). Quantitative elastography, assessed using the fat-to-lesion ratio, was a good discriminant for malignancy (AUROC = $0.93, \mathrm{p}<0.001)$. Our results pointed to an optimal threshold for malignancy of $>4.88$; by using this threshold, the diagnostic reliability of the fat-to-lesion ratio was better than both ultrasonography and qualitative elastography $(86.5 \%$ sensitivity and $90.4 \%$ specificity). Conclusion: Real time elastography is superior to ultrasonography in diagnosing malignant breast nodules. The evaluation of nodules using a fat-to-lesion ratio was a better discriminant for malignancy than qualitative elastography.

Keywords: strain elastography, breast tumor, cut-off strain ratio
\end{abstract}

\section{Introduction}

Accurately diagnosing patients requiring breast surgery by identifying suspicious breast masses is of paramount importance for medical professionals involved in breast pathology. Currently, ultrasonography (US) and mammography are the most frequently used non-invasive methods for detecting breast cancer [13], while Doppler techniques may be used in parallel to provide additional information $[4,5]$ and obtain better diagnostic accuracy [6,7]. Complementary to these methods, elastography adds supplementary information regarding differences in elasticity and stiffness between

Received 24.09.2015 Accepted 07.11.2015

Med Ultrason

2016, Vol. 18, No 2, 195-200

Corresponding author: Bogdan Timar

Department of Medical Informatics

14 A, SP. Tudor Vladimirescu

300173 Timisoara, Romania

Phone: + 40722492892

E-mail: bogdantimar@gmail.com different tissues [8-11]. An increase in tissue stiffness on an elastography scan is usually associated with a malignant tumor [12]. Previous studies have described sensitivities ranging from 0.75 to 0.93 and specificities of up to 0.93 [13-16]; however, these studies had important inter-observer differences [17]. Currently, elastography results are reported either in a qualitative manner as a color map scheme, or from a quantitative approach, with a computer determining the fat-to-lesion ratio (FLR). However, despite the increased sensitivity and specificity of the FLR method, there is no consensus regarding the threshold to differentiate benign and malignant lesions [18].

Our study aimed to evaluate the quality of elastography (both qualitative and quantitative methods) as a complementary method in differentiating the diagnosis of malignant versus benign breast tumors, to compare these results with those from US alone, and based on pathology results, to identify the most appropriate threshold FLR value to differentiate benign and malignant solid breast tumors. 


\section{Material and methods}

The study was performed in accordance with the ethical guidelines of the Helsinki Declaration and was approved by the Ethics Committee of our Center. Written informed consent was obtained from all patients prior any study procedure or data collection

\section{Study patients}

Our prospective study started in January 2014 through to July 2015. Inclusion criteria were: solid breast lesions with complete US evaluation and with pathology report as golden standard diagnostic. Only cases that underwent surgery by the end of the analysis were considered in the final analysis. In these patients, the dominant nodule's characteristics from US and elastography were considered for analysis. Exclusion criteria were considered to be: normal breast US results, cystic lesions, simple fibrocystic mastophatia, subcutaneous lipoma, known malignant breast disease, in any evolution stage.

\section{Breast conventional US, strain elastography}

All cases were evaluated by the ductal breast ultrasound technique [19] performed with a Hitachi Preirus machine (Hitachi Inc., Japan). A special breast probe, EUP- L53L, $92 \mathrm{~mm}$ wide, with water bag device, was used for 2B US. In all cases, the ductal breast evaluation was performed according to Amy's technique [20], radial technique, with the nipple in the left upper corner, peripheral lobar structure in the right of the screen, with all recommended layers in the screen: starting from the skin (upper layer) to the rib structures (lower layer).

A small EUP-L74M, $50 \mathrm{~mm}$ wide, was used for strain elastography evaluation. Two operators, with more than 5 years experience in the field of breast US and elastography, performed all US examinations, using the ductal breast approach, with conventional grey scale 2B US, color Doppler and strain elastography in examinations. The breast evaluation was performed prior to any surgical procedure. The examiners were blinded to the pathology report. The Breast Imaging-Reporting and Data System (BI-RADS) - US criteria were used in the evaluation of each nodule in conventional US mode gray scale and Doppler evaluation [21].

Real time elastography (RTE) was performed after the conventional evaluation, in the same examination session. The probe was positioned perpendicular to the skin during compression. The region of interest (ROI) was set for elastography acquisition; the superior margin included subcutaneous fat, the inferior margin included pectoral muscle and the lateral margin had more than $10 \mathrm{~mm}$ distance from the target lesion. For optimal elastography images, performers obtained images showing either homogeneous color mapping within the ROI or the pressure indicator displayed on the screen, ranging between numbers of 3 and 4 .

The RTE results were recorded in each ultrasound report, separate from the BI-RADS category, regardless of the ultrasound patterns. Color map result and FLR value were noted for each evaluated nodule. Both qualitative and quantitative RTE results were independent from the conventional US studied patterns. All results were recorded. In order to avoid a possible bias generated by inter-observer variations, each patient was evaluated by the same trained and experienced operator for both conventional and RTE ultrasound method. We used color map 1, with red color for soft tissues changing to blue color, for hard lesions. We applied the recommended medium external pressure in order to see the real elastic properties of the compressed tissues [9]. We used the classic Tsukuba scale [13] for the evaluation of the qualitative images. An elastography score (ES) of 4 or 5 was considered to be significant for malignancy.

The fat to lesion ration (FLR) was determined in each nodule. Only scans with proper regions of interest (ROI) were considered. At least two measurements were performed for each solid nodule in radial and antiradial sections. The elasticity scores were documented in the patient reports, but did not influence the BIRADS grading.

\section{Pathology examination}

Each biopsy piece was sent to the pathology department for further analysis after it was formalin-fixed and paraffin embedded. The pathologist on duty prepared the specimen for evaluation. The final analysis was performed by a pathologist specialized in breast diagnosis. The pathologist was blinded to the US and elastography report. Diagnosis was made according to the National Cancer Institute classification [22]. Receptor status evaluation was performed in all cases with a possible malignant diagnosis.

\section{Statistical analysis}

Data were collected and analyzed using SPSS v. 17 (SPSS Inc., Chicago, IL, USA) and are presented as the mean \pm standard deviation (numerical variables with a Gaussian distribution) or the number of cases and percentage of the total (categorical variables). The quality of the diagnostic tests for categorical variables was interpreted using sensitivity, specificity, predictive values (both positive and negative), and accuracy; continuous variables were evaluated using a receiver-operating characteristic (ROC) analysis. The results of RTE report were analyzed separately from the BI-RADS US report. The optimum threshold for a positive diagnosis was calculated according to Youden's method, where the value selected maximizes the sum of the sensitivity and specificity.

In this study, $\mathrm{p}<0.05$ was considered the threshold for statistical significance. 
Med Ultrason 2016; 18(2): 195-200

\section{Results}

Starting January 2014, 1528 ductal breast US examinations were performed at our medical center, from which 428 solid tumors were identified; 715 cases had normal US report (BIRADS-US 1), 311 cases had simple unique or multiple cystic lesions, 29 cases had subcutaneous lipomas, 35 had skin lesion, with no breast tissue involvement, 10 cases had isolated linear calcifications, and 317 had surgical indications (malignancy suspicion, dimensions, pain, cosmetic reasons).

Additional magnetic resonance imaging (MRI) or mammography studies were performed in selected cases; however, since these were not part of our study aims, they are not presented in this paper. Through the end of the follow-up period (July 2015), 174 patients underwent surgery from the total of 317 patients referred to the surgery service. In these patients, the diagnostic nodule's characteristics from US and elastography were considered for analysis. These cases were comprised in the final analysis.

A total of 174 patients (mean age, $46.5 \pm 11.3$ years; range, 18 to 79 years) underwent surgery: 102 patients had benign lesions (58.6\%) and $72(41.37 \%)$ breast cancer. The majority of the observed nodules were $<25$ $\mathrm{mm}$ (146 cases, 83.90\%). We evaluated the following malignant characteristics, as recommended by the BIRADS - US criteria [21]: round shape $(25.3 \%)$ versus irregular/spiculated/atypical shapes $(29.7 \%)$, vertical dominant axis (41.4\%), irregular, not circumscribed margins: (26.4\%), abrupt interfaces with the surrounding tissue $(38.5 \%)$, heterogeneous patterns $(14.9 \%)$, and the presence of micro-calcifications, defined as discontinuous echogenic spots within a mass or hypoechoic area, or clustered echogenic spots clearly different from the mammary glands $(38.5 \%)$, as described as in the literature for Hitachi Preirus ultrasound devices [23].

According to this, risk stratification for malignancy was made for conventional BIRADS - US classification, gray scale and Doppler results, respectively, color map $1-2,3$ and 4-5 results, as presented in Table I.
Table I. Risk stratification of the operated lesions $(n=174)$ by means of conventional ultrasonography, 2B plus color Doppler evaluation, and real time elastography.

\begin{tabular}{llll}
\hline Technique & $\begin{array}{l}\text { Low } \\
\text { risk }\end{array}$ & $\begin{array}{l}\text { Intermediate } \\
\text { risk }\end{array}$ & $\begin{array}{l}\text { High } \\
\text { risk }\end{array}$ \\
\hline 2B US & 105 & 61 & 6 \\
2B US + Doppler & 99 & 69 & 9 \\
RTE - Tsukuba scale & 91 & 58 & 25 \\
ES 1-2, 3, 4-5 & & &
\end{tabular}

2B US - bidimensional ultrasonography, RTE - real time elastography, ES - elastography score

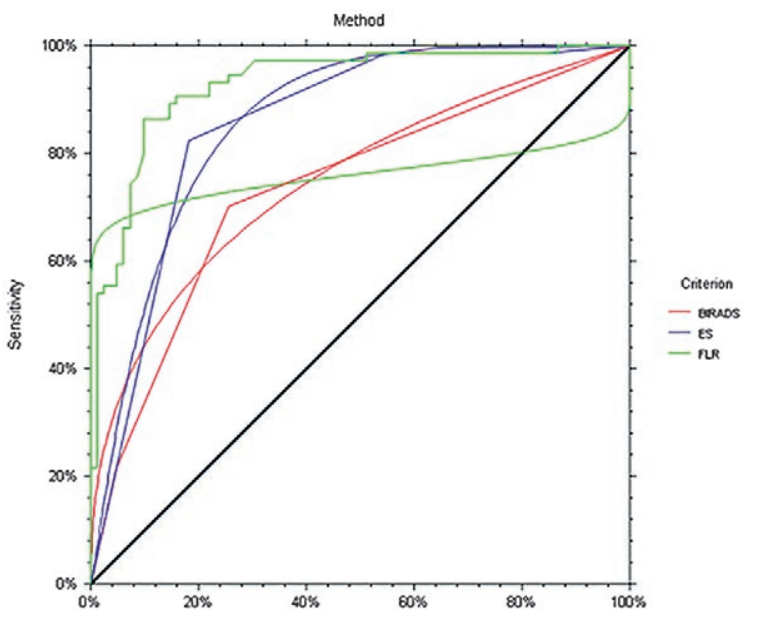

Fig 1. Receiver-operating characteristic curve using the conventional ultrasound evaluation, qualitative (color map elastography), and fat-to-lesion ratio as a diagnostic tool for malignant breast nodules. BIRADS $=$ conventional ultrasound (2B and Doppler) result, $\mathrm{ES}=$ color map elastography, $\mathrm{FLR}=$ fat to lesion ration.

The diagnostic characteristics of US techniques, compared with the pathology report results are detailed in Table II.

The diagnostic qualities of the different diagnostic methods are presented in fig 1 . The significance difference in diagnostic power is seen in the area under ROC curve: conventional US (gray scale and Doppler scan) $\mathrm{AUC}=73.38 \%$, [95\% CI $65.33 \%$ to $79.79 \%$ ], $\mathrm{p}<0.001$; qualitative strain elastography $\mathrm{AUC}=85.25 \%[95 \% \mathrm{CI}$,

Table II. Comparisons between diagnostic performances of different ultrasound techniques

\begin{tabular}{llllll}
\hline & Sensitivity (\%) & Specificity (\%) & PPV (\%) & NPV (\%) & Accuracy (\%) \\
\hline 2B US & 62.1 & 66.6 & 50.72 & 64.76 & 65.51 \\
2B US + Doppler & 70.3 & 73.5 & 70.3 & 73.5 & 71.9 \\
Qualitative RTE & 82.4 & 81.9 & 80.3 & 83.9 & 82.2 \\
$\begin{array}{l}\text { Tsukuba scale ES 4-5) } \\
\text { Quantitative }\end{array}$ & 86.5 & 90.4 & 88.9 & 88.2 & 88.5 \\
RTE FLR $(>4.88)$ & & & &
\end{tabular}

2B US - bidimensional ultrasonography, RTE - real time elastography, FLR - fat to lesion ratio, PPV - positive predictive value, NPV negative predictive value 
$78.26 \%$ to $90.12 \%$ ], $\mathrm{p}<0.001$ and quantitative strain elastography, AUC $=93.029 \%$ [95\% CI $87.60 \%$ to $96.12 \%$ ], $\mathrm{p}<0.001$.

RTE increased the sensitivity and specificity of the conventional US evaluation. We found that the best threshold value for the FLR to differentiate malignant lesions, calculated according to Youden's method is 4.88. If 4.88 were considered the threshold, the FLR measurement method demonstrates an improved diagnostic performance when compared with ultrasonography and qualitative elastography, having a sensitivity of $86.5 \%$ and a specificity of $90.4 \%$. The values are calculated for the prevalence of cancer in the study group, of $41.37 \%$

The lesion size did not have any impact of elastography results. If we consider the calculated FLR cutoff point of 4.88 , in lesions $<25 \mathrm{~mm}$ the accuracy of the method was $93.15 \%(136 / 146$ cases $)$ and in lesions $>25$ $\mathrm{mm}$ the accuracy was $92.85 \%$ (26/28 cases).

For elastography, the inter-observer agreement was substantial, with a K coefficient of 0.68 .

\section{Discussions}

Breast cancer is the most frequent cancer, affecting females worldwide [24]. Furthermore, despite the decrease in prevalence in some European countries, our country has shown a sustained increase [25]. On average, in our country breast cancer is diagnosed at a younger age (44.3 years) comparing with patients in other Eastern European countries such as Hungary (63.0 years), Croatia (55.8 years), Serbia (57.9 years), and Poland (44.5 years) [26]; additionally, Romania has the highest cancer mortality rate $(30.9 / 100,000)$.

There is intense debate regarding the optimal screening approach for breast cancer. Even if mammography techniques are used as often as recommended, their applicability is limited by dense breast tissue, which is described in almost all premenopausal breasts and in women younger than 50 years [27]. There are also fears of the pain and irradiation produced by mammography in the Romanian female population. The lack of addressability of early detection methods is considered to be responsible for the large number of patients diagnosed in more advanced stages [28].

Breast US diagnostic is a non-irradiating evaluation, with no pain or discomfort for the patient. Ductal approach allows a better identification and localization of even small solid lesions, and provides a good description of their relation to the surrounding tissues and connecting structures. The rate of acceptance of this method is high in the female population; however, the moderate sensitivity and specificity of this method, also confirmed by the results of our study (both approximately $70 \%$ ), leads to some false results (both positive and negative).

RTE is a newer method with increased diagnostic quality compared with conventional ultrasonography alone and allows for two possible approaches: a qualitative approach using color scales (in our case, the Tsukuba scale), in which the subjectivity of the investigator could be a possible bias, and a quantitative, objective approach, which consists of evaluating the FLR. The literature has cited significant increases in sensitivity, from $78 \%$ to $91.5 \%$ [29] or from $71.2 \%$ to $89.7 \%$ [16]; our results revealed similar increases [30].

After we performed RTE, we reclassified 48 cases as follows: 25 negatives at US were classified as positives (of which 16 were true positives and 9 were false positives from the pathology findings) and 23 positives were classified as negatives (of which 16 were true negatives and 7 false negatives from the pathology findings). The difference in specificity was most probably caused by false negative results in the color map mode: borderline lesions that are too hard to be a plain $\mathrm{ES}=3$ score on the color map, but not hard enough for a score of 4 . Some authors have stated that benefits of the RTE technique are seen in cases that have benign US characteristics, are classified as BI-RADS - US 3, and show increased hardness on elastography [30] and thus are reclassified in the BI-RADS - US 4 category [31].

Other studies showed better results for the FLR (qualitative technique) calculation than for the color map evaluation [29]; the use of a computer-assisted quantitative technique, especially in borderline cases on a color map scheme (qualitative technique), where the subjectivity of the operator can influence the results is needed.

The threshold value that should be used is still an open question $[18,33]$. There is no recommended unique value, neither for any type of elastography or for a specific manufacturer. Most authors have used values around 4 and 5: 4.8 [13], 4.7 [24], 4.0 [31], 4 to 5 [32], respectively 4.18 [34]. However, other authors have recommended a value of 3 or less as a good threshold point: 2.90 [35], 3.8 [36], 3.64 [37], 3.5 [38], 3 [32], and 2.61 [39]. There are also some authors that recommend higher cut-off values, up to 5.6 [40]. Also, some authors identified a specific threshold for malignant lesions, $3.04 \pm 0.9$ with suggestive values for benign lesions, $1.91 \pm 0.75$ [41].

In our case, the FLR proved to have both better sensitivity and specificity for the diagnosis of malignant lesions than ultrasonography and qualitative RTE. According to our results, the best threshold value for malignancy is an FLR $>4.88$. There is still no general agreement regarding which threshold value should be used [33] but the overall sensitivity and specificity of RTE are excel- 
lent [42], quantitative techniques appearing to be better than the qualitative technique [43].

\section{Limits of the study}

The percentage of patients that were compliant to the further evaluation recommendation was relatively small (174 out of 317 cases) so the real incidence of malignant lesions in the evaluated group is underestimated. As in all RTE studies, the value of FLR threshold is debatable. Different thresholds will deliver different diagnostic qualities. Ideally a recommended FLR Ratio, for each RTE device should be used in order to make head to head comparisons between different studies.

\section{Conclusions}

RTE is superior to conventional and Doppler ultrasonography in the differentiation of benign and malignant solid breast masses. A quantitative computer-assisted stiffness evaluation demonstrated a better diagnostic performance than the color map RTE. Standardized threshold values, validated for each elastography device, should be proposed and applied in order to provide a more accurate differential diagnosis between malignant and benign breast nodules. Integrating the elastography information in the complete breast ultrasound evaluation adds diagnostic quality.

Acknowledgement: This paper is part of the research grant: SMIS 45997/21.01.2014- "Crestera calitatii actului medical prin valorificarea potentialului IT" Guvernul Romaniei, Ministerul Comunicatiilor si societatii informationale, axa POS CCE.

Conflict of interest: none

\section{References}

1. Stavros AT, Thickman D, Rapp CL, Dennis MA, Parker SH, Sisney GA. Solid breast nodules: use of sonography to distinguish between benign and malignant lesions. Radiology 1995; 196: 123-134.

2. Jackson VP. The current role of ultrasound in breast imaging. Radiol Clin North Am 1995; 33: 1161-1170.

3. Merz E, Oberstein A. Breast ultrasound. In: Merz E. U1trasound in obstetrics and gynecology. Stuttgart: Thieme, 2007: 265-296.

4. Hayashi N, Miyamato Y, Nakata N, et al. Breast masses: color Doppler, power Doppler and spectral analysis findings. J Clin Ultrasound 1998; 26: 231-238.

5. Kook SH, Park HW, Lee YR, Lee YU, Pae WK, Park YL. Evaluation of solid breast lesions with power Doppler sonography. J Clin Ultrasound 1999; 27: 231-237.

6. Lee SW, Choi HY, Baek SY, Lim SM. Role of color and power Doppler imaging in differentiating between malig- nant and benign solid breast masses. J Clin Ultrasound 2002; 30: 459-464.

7. Tozaki M, Fukuma E. Does power Doppler ultrasonography improve the Bi-RADS category assessment and diagnostic accuracy of solid breast lesions? Acta Radiol 2011; 52: 706-710.

8. Hiltawasky KM, Kruger M, Starke C, Heuser L, Ermert $\mathrm{H}$, Jensen A. Freehand ultrasound elastography of breast lesions: clinical results. Ultrasound Med Biol 2001; 27: 1461-1469.

9. Itoh $\mathrm{A}$, Ueno $\mathrm{E}$, Tohno E, et al. Breast disease: clinical application of US elastography for diagnosis. Radiology 2006; 239: 341-350.

10. Zhi H, Xiao XY, Ou B, et al. Could ultrasonic elastography help the diagnosis of small $(\leq 2 \mathrm{~cm})$ breast cancer with the usage of sonographic BI-RADS classification? Eur J Radiol 2012; 81: 3216-3221.

11. Stoian D, Cornianu M, Dobrescu A, Lazăr A. Nodular thyroid cancer. Diagnostic value of real time elastography. Chirurgia (Bucur) 2012; 107: 39-46.

12. Barr RG. Sonographic breast elastography. J Ultrasound Med 2012; 31: 773-783.

13. Ueno E, Umemoto T, Bando H, Tohno E, Waki K, Matsumura T. New quantitative method in breast elastography: fat-lesion ratio (FLR) [abstract]. In: Proceedings of the Radiological Society of North America Scientific Assembly and Annual Meeting. Oak Brook, IL: Radiological Society of North America; 2007: 697.

14. Wojcinski S, Farrokh A, Weber S, et al. Multicenter study of ultrasound real-time tissue elastography in 779 cases for the assessment of breast lesions: improved diagnostic performance by combining the BI-RADS $\AA-U S$ classification system with sonoelastography. Ultraschall Med 2010; 31: 484-491.

15. Raza S, Odulate A, Ong MW, Chikarmane S, Harston CW. Using real-time tissue elastography for beast lesion evaluation: our initial experience. J Ultrasound Med 2010; 29: 551-563.

16. Zhi H, Ou B, Luo BM, Feng X, Wen YL, Yang HY. Comparison of ultrasound and elastography, mammography, and sonography in the diagnosis of solid beast lesions. J Ultrasound Med 2007; 26: 807-815.

17. Yoon JH, Kim MH, Kim EK, Moon HJ, Kwak JY, Kim MJ. Interobserver variability of US elastography: how it affects the diagnosis of breast lesions.AJR Am J Roentgenol 2011; 196: 730-736.

18. Cosgrove D, Piscaglia F, Bamber J, et al. EFSUMB guidelines and recommendations on the clinical use of ultrasound elastography. Part 2: Clinical applications. Ultraschall Med 2013; 34: 238-253.

19. Amy D. Ecographie mammaire: echoanatomie. J1 mensuele d'ecographie. LUS 2000; 10: 654-662.

20. Amy D. Lobar breast ultrasound. In Tibor Tot (eds.) Breast Cancer - a lobar disease. London Dordrecht Heidelberg New York. Springer 2011: 153-162.

21. American College of Radiology (ACR) Breast Imaging Reporting and Data System Atlas (BI-RADS Atlas). Reston, Va: C American College of Radiology; 2003. 
22. Breast. In: Edge SB, Byrd DR, Compton CC, et al, (eds.). AJCC Cancer Staging Manual. $7^{\text {th }}$ ed. New York, NY: Springer, 2010: 347-376.

23. Hashimoto Y, Murata A, Miyamoto N, et al. Clinical significance of microcalcifications detection in invasive breast carcinoma. Yonago Acta Med 2015; 58: 89-93.

24. Bray F, McCarron P, Parkin DM. The changing global patterns of female breast cancer incidence and mortality. Breast Cancer Res 2004; 6: 229-239.

25. Znaor A, van den Hurk C, Primic-Zakelj M, et al. Cancer incidence and mortality patterns in South Eastern Europe in the last decade: gaps persist compared with the rest of Europe. Eur J Cancer 2013; 49: 1683-1691.

26. Vrdoljak E, Wojtukiewicz MZ, Pienokowski T, et al; South Eastern European Research Oncology Group. Cancer epidemiology in Central and South Eastern European countries. Croat Med J 2001; 52: 478-487.

27. Bird RE, Wallace TW, Yankaskas BC. Analysis of cancers missed at screening mammography. Radiology 1992; 184: 613-617.

28. Lucaci L, Szucsik IA. Statistical study of the incidence and prevalence of breast cancer in Arad County, between the years 1999-2009. Arad Medical Journal 2010: XIII: 5-10.

29. Chung SY, Moon WK, Choi JW, Cho N, Jang M, Kim KG. Differentiation of benign from malignant nonpalpable breast masses: a comparison of computer-assisted quantification and visual assessment of lesion stiffness with the use of sonographic elastography. Acta Radiol 2010; 51: 9-14.

30. Stoian D, Craina M, Craciunescu M. Diagnostic value of real-time elastography in apparent benign lesions (abstract). The Breast 2013; 22 (Supple 1): S 45.

31. Wojcinski S, Boehme E, Farrokh A, Soergel P, Degenhardt F, Hillemanns P. Ultrasound real-time elastography can predict malignancy in BI-RADS(R)-US 3 lesions. BMC Cancer $2013 ; 13: 159$.

32. Venugopal V, Ahmad I, Afshan I. Quantitative elastographic assessment of nonpalpable breast nodules by measuring fat-lesion strain-ratio vs qualitative color elastography scores: comparison of diagnostic performances by blinded prospective study. Radiological Society of North America 97 $7^{\text {th }}$ Scientific Assembly and Annual Meeting. November $27^{\text {th }}$ December $2^{\text {nd }}, 2011$, Chicago, USA.

33. Barr RG, Nakashima K, Amy D, et al. WFUMB guidelines and recommendations for clinical use of ultrasound elastography: Part 2: Breast. Ultrasound Med Biol 2015; 41: 1148-1160.
34. Yi A, Cho N, Chang JM, Koo HR, La Yun B, Moon WK. Sonoelastography for 1786 non-palpable breast masses: diagnostic value in the decision to biopsy. Eur Radiol 2012; 22: 1033-1040.

35. Ebner L, Bonel HM, Christe A; Bern/CH. Is real-time elastography (RTE) a valuable addition to morphologic ultrasound for differentiating benign and malignant breast tumors? ECR 2013, March 8th - 11th, Vienna, Austria. Hitachi Real-time Tissue Elastography for Women's Health 2013. Hitachi Real-time Tissue Elastography: Publications \& International communications. Clinical Abstracts. Accessible on: http://tinyurl.com/ hIRTE-bibliography.

36. Farrokh A, Wojcinski S, Degenhardt F. Diagnostic value of strain ratio measurement in the differentiation of malignant and benign breast lesions. Ultraschall Med 2011; 32: 400-405.

37. Gheonea IA, Stoica Z, Bondari S. Differential diagnosis of breast lesions using ultrasound elastography. Indian J Radiol Imaging 2011; 21: 301-305.

38. Ebner L, Bonel HM, Christe A. Is real-time elastography a value addition to morphologic ultrasound for differentiating benign and malignant breast tumors? Paper presented at: European Congress of Radiology. $8^{\text {th }}-11^{\text {th }}$ March 2013, Vienna, Austria.

39. Dobruch-Sobczak I, Sudol-Szopinska I. The usefulness of sonoelastography in the differential diagnosis of solid breast lesions. Ultrasound Med Biol 2011; 37: S100.

40. Alhabshi SM, Rahmat K, Abdul Halim N, et al. Semi-quantitative and qualitative assessment of the breast ultrasound elastography in differentiating between malignant and benign lesions. Ultrasound Med Biol 2013; 39: 568-578.

41. Stachs A, Hartmann S, Stubert J, et al. Differentiating between malignant and benign breast masses: factors limitating sonoelastographic strain ration. Ultraschall Med 2013; 34: 131-136.

42. Sadigh G, Carlos RC, Neal CH, Dwamena BA. Accuracy of quantitative ultrasound elastography for differentiation of malignant and benign breast abnormalities: a meta-analysis. Breast Cancer Res Treat 2012; 134: 923-931.

43. Ebner L, Bonel HM, Huber A, Ross S, Christe A. Diagnostic Performance and Additional Value of Elastosonography in Focal Breast Lesions: Statistical Correlation between Size-Dependant Strain Index Measurements, Multimodality-BI-RADS Score, and Histopathology in a Clinical Routine Setting. ISRN Radiol 2014; 2014: 396368. 